JOURNAL OF SYNCHROTRON RADIATION

ISSN 1600-5775

Received 27 February 2019

Accepted 11 November 2019

Edited by P. A. Pianetta, SLAC National Accelerator Laboratory, USA

Keywords: cryogenic soft X-ray tomography; cryogenic fluorescence microscopy; precise alignment; correlative imaging method; organelle identification.

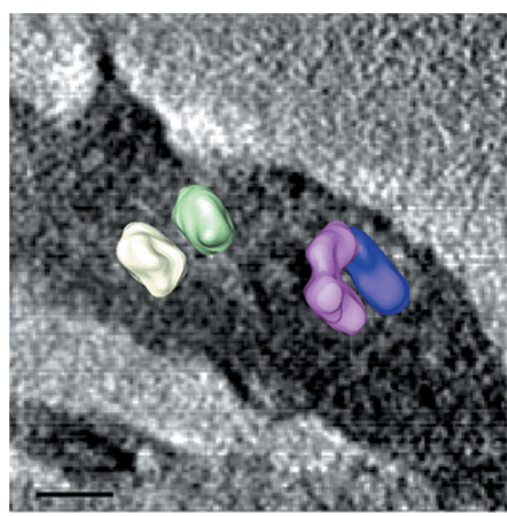

C 2020 International Union of Crystallography

\section{Precise correlative method of Cryo-SXT and Cryo-FM for organelle identification}

\author{
Haobo Bai, Yong Guan,* Jianhong Liu, Liang Chen, Wenbin Wei, Gang Liu and \\ Yangchao Tian*
}

\begin{abstract}
National Synchrotron Radiation Laboratory, University of Science and Technology of China, No. 42 Hezuohua South Road, Hefei, Anhui 230029, People's Republic of China. *Correspondence e-mail: yongg@ustc.edu.cn, ychtian@ustc.edu.cn
\end{abstract}

Cryogenic soft X-ray tomography (Cryo-SXT) is ideally suitable to image the $3 \mathrm{D}$ sub-cellular architecture and organization of cells with high resolution in the near-native preservation state. Cryogenic fluorescence microscopy (Cryo-FM) can determine the location of a molecule of interest that has been labeled with a fluorescent tag, thus revealing the function of the cells. To understand the relations between the sub-cellular architecture and the function of cells, correlative Cryo-SXT and Cryo-FM was applied. This method required the matching of images of different modalities, and the accuracy of the matching is important. Here, a precise correlative method of Cryo-SXT and Cryo-FM is introduced. The capability of matching images of different modalities with high resolution was verified by simulations and practical experiments, and the method was used to identify vacuoles and mitochondria.

\section{Introduction}

Cryogenic soft X-ray tomography (Cryo-SXT) is a rapidly developing high-resolution 3D imaging technology used to reveal sub-cellular architecture and organization in the nearnative state. In the region of the spectrum that lies between the $K$-shell absorption edges of carbon $(284 \mathrm{eV}, \lambda=4.4 \mathrm{~nm})$ and oxygen ( $543 \mathrm{eV}, \lambda=2.3 \mathrm{~nm})$, called the 'water window', photons are absorbed an order of magnitude more strongly by carbon- and nitrogen-containing organic material than by water. Thus, soft X-ray microscopy is able to penetrate mesoscale biological specimens up to $10 \mu \mathrm{m}$ with high contrast (Wolter, 1952; McDermott et al., 2009). The spatial resolution of soft X-ray imaging is as low as $15 \mathrm{~nm}$ (Chao et al., 2005). Using tomography, soft X-ray microscopy can facilitate the $3 \mathrm{D}$ reconstruction of a specimen by taking a series of $2 \mathrm{D}$ projection images from different angles. During collection of these images, biological samples are fixed in ice to avoid any movement that could cause details in images to blur and to reduce radiation damage (Hagen et al., 2012; Schneider, 1998; Carzaniga et al., 2014).

To understand interconnected molecular interactions and chemical reactions in cells, knowledge of only the sub-cellular architecture and organization is not sufficient (McDermott et al., 2009). As a vital complementary technology, cryogenic fluorescence microscopy (Cryo-FM) can locate molecules that are essential for cell functions. Correlative Cryo-SXT and Cryo-FM, integrating both the sub-cellular architecture and the information of specific molecules within one framework, can help to elucidate the relationship between cellular structure and function and thus is a useful technology for research in cell organelle discrimination, pathopoeia and drug therapy mechanisms. 
Organelles exist in various types of cells including animals, plants and bacteria, and perform necessary functions for normal cellular activities; they are important parts of cells (Smith, McDermott et al., 2014; Elgass et al., 2015; Larabell \& Le Gros, 2004; Walters et al., 2014). However, several types of organelles exist, thus making their discrimination indispensable. Organelles can be distinguished by morphological features, linear absorption coefficient (LAC) values or different fluorescence labels. However, some organelles have similar morphological features or LAC values. Methods using fluorescence labels have been developed in which most organelles can be specifically labeled by a fluorescence reagent. By combining the sub-cellular architecture and the organization information offered by Cryo-SXT, a particular organelle can be distinguished from other organelles, and the relation between the organelles and other parts of the cell can be obtained.

Images of different modalities need to be precisely matched for identification of organelles by the correlation method. Two methods of matching images of different modalities exist. (i) Locating images of the same object in different modalities by directly observing the similarities between soft X-ray and fluorescence images (McDermott et al., 2009, 2012; Smith et al., 2013; Sorrentino et al., 2015; Duke et al., 2014). This method is straightforward, but its accuracy is relative to the sizes of the images of the object and is influenced by subjectivity. (ii) Using fiducial markers. Images of different modalities are aligned by manually locating fiducial markers. Then, the position of the object is correlated with its fluorescence marker. Common fiducial markers are stained organelles (Smith et al., 2013; Smith, Cinquin et al., 2014; Smith, McDermott et al., 2014; Elgass et al., 2015; Do et al., 2015; Ekman et al., 2017; Varsano et al., 2016, 2018; Cinquin et al., 2014) and fluorescence spheres (Hagen et al., 2012; Zeev-Ben-Mordehai et al., 2014). The accuracy of the location depends on the size of the fiducial markers: the smaller the fiducial marker, the higher the location accuracy. However, this accuracy cannot be better than the spatial resolution of fluorescence microscopy. The locating accuracy of the second method can be good enough when using super-resolution fluorescence microscopy, because this resolution is close to that of soft X-ray microscopy (Varsano et $a l ., 2016)$. For wide-field microscopy and confocal microscopy, the locating accuracy can be further improved.

If an object is much smaller than the spatial resolution of the fluorescence, it can be treated as a single 'point-like' particle; then the center of the object can be located precisely by algorithms, and the accuracy can be much better than the spatial resolution of fluorescence microscopy (Cheezum et al., 2001; Thompson et al., 2002). Furthermore, if the object is visible in both microscopy types, it can be used as a fiducial marker (Schellenberger et al., 2014). As long as there are enough fiducial markers in the region of interest, the object can be located precisely using images obtained by both microscopies.

In this study, we used $200 \mathrm{~nm}$ fluorescence spheres as fiducial markers, having high fluorescence intensity and X-ray microscope contrast. The locating accuracy can be very high if the markers are isolated. MATLAB scripts were used to locate these fiducial markers and match the Cryo-SXT and Cryo-FM images. Simulations and experiments were performed to verify the locating accuracy of this proposed method. The results show that the total locating accuracy is better than $200 \mathrm{~nm}$. Vacuoles of yeast and mitochondria were located using this method. The results show that the proposed method can be useful for recognition and classification of organelles.

\section{Method and materials}

The correlative method of Cryo-SXT and Cryo-FM consists of two parts - alignment and fusion (Dent et al., 2014; Duke et al., 2014; Smith et al., 2013). The alignment between images of different modalities can eliminate the differences of scales and locations between corresponding objects in the images. Then, the corresponding images are fused to integrate the information of the different modalities. The relations between subcellular architecture and specific molecules can be easily observed in the fused image.

The alignment method can be divided into three parts. First, a rough region containing the fiducial markers is captured manually as a series of small images. The correspondence between the fiducial markers of different modalities is obtained, and the fiducial markers are located in soft $\mathrm{X}$-ray projections and fluorescence images by calculating their position with these small images. These images are divided into object pixels and background pixels using the Otsu threshold method, and then the centers of the fiducial markers are calculated as object-pixel-value-weighted object-pixel locations. For the soft X-ray projections, darker pixel values, corresponding to the heavier absorption of X-rays, have higher weights; for the fluorescence images, the lighter pixel values, corresponding to stronger fluorescence intensity, have higher weights. An iterative method is applied to improve the accuracy. Location accuracies $\sigma_{\mathrm{X}}$ (the accuracy of locating fiducial markers in the X-ray projections) and $\sigma_{\mathrm{F}}$ (the accuracy of locating fiducial markers in the fluorescence images) are calculated from the standard deviation of several computed results. Lastly, X-ray projections and fluorescence images are matched based on the fiducial markers. Fluorescence images should be transformed before being matched with X-ray projections. The transform parameters can be calculated using the positions of two sets of corresponding fiducial markers, for which at least two fiducial markers in each modality are required. For calculating the alignment accuracy $\sigma_{\mathrm{A}}$, more than two fiducial markers in each modality are needed (Schellenberger et al., 2014). Within the calculation, a pair of fiducial markers are excluded to calculate the transform parameters of other fiducial markers. Then, the error can be obtained by comparing one excluded fiducial marker and the transformation of its counterpart. This step is repeated for all fiducial markers in the images, and the mean value of the displacements provides an estimate of the alignment accuracy $\sigma_{\mathrm{A} 1}$. Another method has been applied to calculate the alignment accuracy $\sigma_{\mathrm{A}}$ (Mohammadian et al., 2019). Positions of fiducial markers in the fluorescence image were trans- 
formed to positions in the soft X-ray image, then the average distance between these positions and the positions of corresponding fiducial markers in the soft X-ray image located automatically was calculated as the alignment accuracy $\sigma_{\mathrm{A} 2}$. Finally, if $\sigma_{\mathrm{X}}, \sigma_{\mathrm{F}}$ and $\sigma_{\mathrm{A}}$ are treated as being independent, the overall uncertainty is given by $\sigma_{\mathrm{T}}=\left(\sigma_{\mathrm{X}}^{2}+\sigma_{\mathrm{F}}^{2}+\sigma_{\mathrm{A}}^{2}\right)^{1 / 2}$ (Schellenberger et al., 2014). After alignment of the images, the images are fused by an algorithm based on the HSI (hue, saturation, intensity) color space. This takes advantage of the precise alignment result and automatically fuses images of different modalities. From the fused image, the relations between different modalities can be clearly observed.

\subsection{Simulation}

To validate our correlative method, simulations and experiments were performed.

A 3D Shepp-Logan phantom with fiducial markers at the bottom of the phantom was generated. The locations of these fiducial markers were chosen from a few random sets to avoid too much agglomeration between them. In general, fiducial markers are preferably isolated, and there must be at least two of them in the field of view. The accuracy can be calculated with more than two fiducial markers. In addition, it is better that the largest distance between fiducial markers is larger than $7 \mu \mathrm{m}$. Then, the soft X-ray projection and fluorescence images at different focal planes are generated (Fig. 1). The simulated soft X-ray projection consisted of $950 \times 950$ pixels with a pixel size of $15.8 \mathrm{~nm}$, and the fluorescence images consisted of $171 \times 171$ pixels with a pixel size of $88 \mathrm{~nm}$. A 3D point spread function of the fluorescence was generated by a fast and accurate approximation of the Gibson-Lanni model with a full width at half-maximum (FWHM) of $440 \mathrm{~nm}$ (Li et al., 2017) [parameters of the model: magnification factor, 50; oversampling ratio, 6; numerical aperture, 0.6; emission wavelength in vacuum, $510 \mathrm{~nm}$; specimen refractive index (RI), 1.33; designed coverslip RI, 1.5; experimental coverslip RI, 1.5; designed immersion RI, 1; experimental immersion RI, 1; designed working distance, $4 \mathrm{~mm}$; designed coverslip thickness, $170 \mu \mathrm{m}$; experimental coverslip thickness, $170 \mu \mathrm{m}$; position of particle, $4 \mu \mathrm{m}$ ]. If some of the fluorescence spheres were too close to be distinguished by the fluorescence images [Fig. 1(b)], they were regarded as one fiducial marker throughout the whole simulation.

Then, the positions of the fiducial markers were located, and the accuracies of locating using the algorithm or manually locating the markers were measured. If a position contains more than one fluorescence sphere, the location value of these spheres will be calculated as the center of the fluorescence signal or soft X-ray projection spots. The fiducial markers were manually located as described earlier, and the average distances between them were calculated and the corresponding actual locations as manual locating accuracies. Automatically locating the fiducial markers required rough positions of the fiducial markers as the initial positions. The actual locations and manual locating results were used as initial positions to measure uncertainties caused only by the
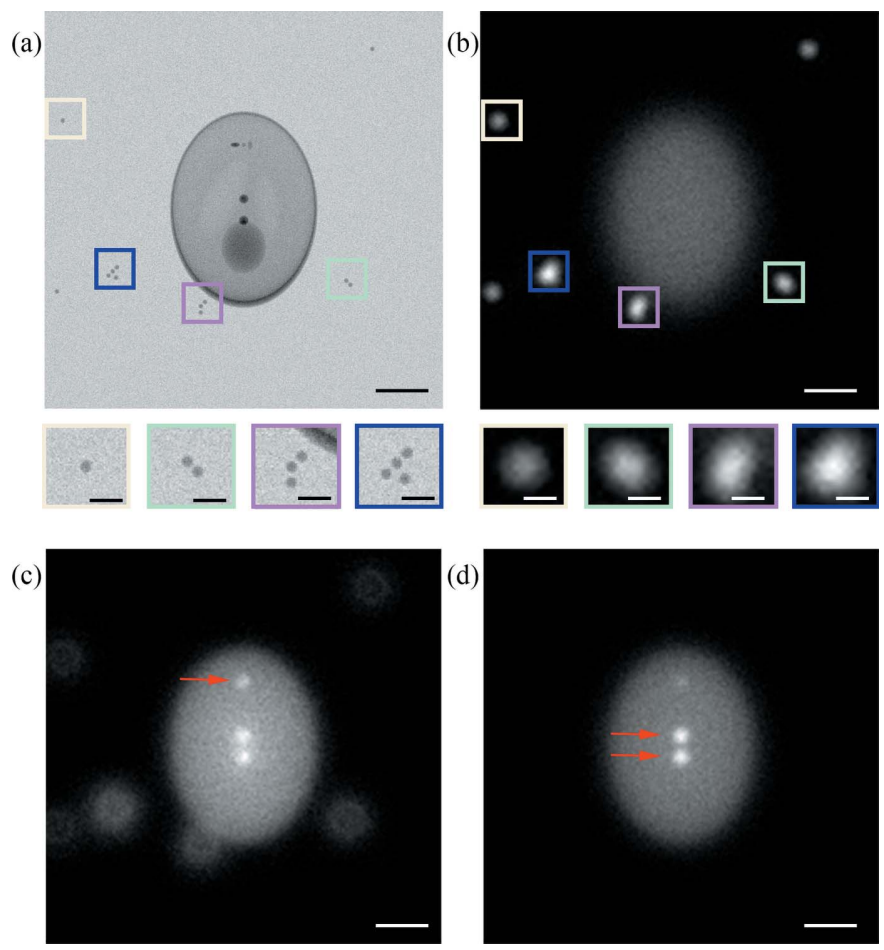

Figure 1

(a) Simulated soft X-ray projection and $(b)-(d)$ fluorescence images with random fluorescence spheres. The small images at the bottom of $(a)$ and (b) show situations in which a few fluorescence spheres agglomerate in the soft X-ray projection and fluorescence image. (b) Fluorescence spheres and $(c)-(d)$ stained structures indicated by red arrows are in the focal planes. Scale bars: $(a)-(d): 2 \mu \mathrm{m}$; small images at the bottom of $(a)$ and (b): $500 \mathrm{~nm}$.

program and caused by the program with manual locating results. Locating accuracies are the average distances between the automatically located results and the corresponding actual locations. Each accuracy was the average of results of ten independent simulations. The results are shown in Fig. 2.

For exploring the program dependence on the manual locating results, we measured the automatic locating accuracies using the manual locating results as the initial position (shown in Fig. 3). The distance between the initial positions

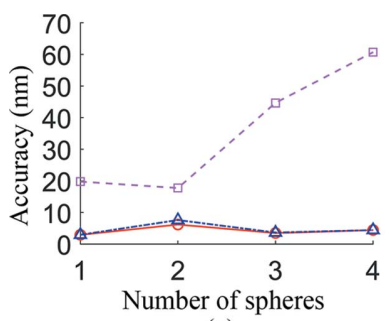

(a)

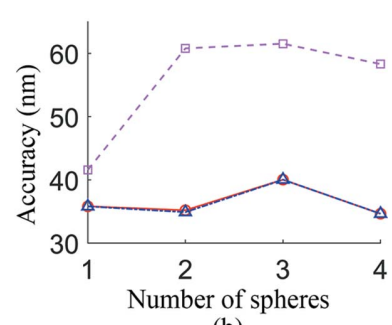

(b)
Figure 2

Locating accuracies were calculated using different methods. The results of the simulated X-ray projections are shown in $(a)$ and the results of the simulated fluorescence images are shown in $(b)$. Horizontal axes represent the numbers of fluorescence spheres contained in each fiducial marker. The purple lines with squares, red lines with circles and blue lines with triangles represent the accuracies of locating manually, locating automatically using actual positions and locating automatically using manual locating results as initial positions, respectively. 
and corresponding fiducial markers gradually increased, while the automatic locating accuracies were stable at a certain distance and sharply increased after that.

The automatic locating accuracies of the fiducial markers were less than $10 \mathrm{~nm}$ for the X-ray projection and less than $40 \mathrm{~nm}$ for the fluorescence images, no matter which actual locations or manual locating results were used as initial positions and no matter if a fiducial marker contained one or more fluorescence spheres. Locating accuracies attained sub-pixel values both in X-ray projections and fluorescence images. This demonstrates that our program is capable of locating fiducial markers with high accuracies. In contrast, manual locating accuracies were 20-60 nm for X-ray projections and 40-60 nm for fluorescence images. When manual locating results were used as the initial position, the locating program was almost uninfluenced by the manual uncertainty. Although the locating program failed when the center of the manuallocating result was far from the actual position, this could be avoided because it only happened when the distance was more than $200 \mathrm{~nm}$ for the X-ray projection and more than $1800 \mathrm{~nm}$ for the fluorescence image, where the diameter of the fluorescence sphere was $200 \mathrm{~nm}$. This demonstrates that our program could exclude the subjective influence of manual operation, although manual locating is used as the first step.

The alignment accuracies with 20 independent sets of points, whose locating accuracies were the same as ones detected in previous simulations, were calculated. The average alignment accuracy $\sigma_{\mathrm{A} 1}$ calculated by the algorithm was $22.8 \mathrm{~nm}$, and the average alignment accuracy $\sigma_{\mathrm{A} 2}$ was $17.5 \mathrm{~nm}$. The average total accuracy $\sigma_{\mathrm{T}}$ can be calculated using the formula $\sigma_{\mathrm{T}}=\left(\sigma_{\mathrm{X}}^{2}+\sigma_{\mathrm{F}}^{2}+\sigma_{\mathrm{A}}^{2}\right)^{1 / 2}$, where $\sigma_{\mathrm{T} 1}$ corresponding to $\sigma_{\mathrm{A} 1}$ was $44.3 \mathrm{~nm}$ and $\sigma_{\mathrm{T} 2}$ corresponding to $\sigma_{\mathrm{A} 2}$ was $41.8 \mathrm{~nm}$.

A simulated X-ray projection and the simulated fluorescence images were fused with the total accuracy, which was

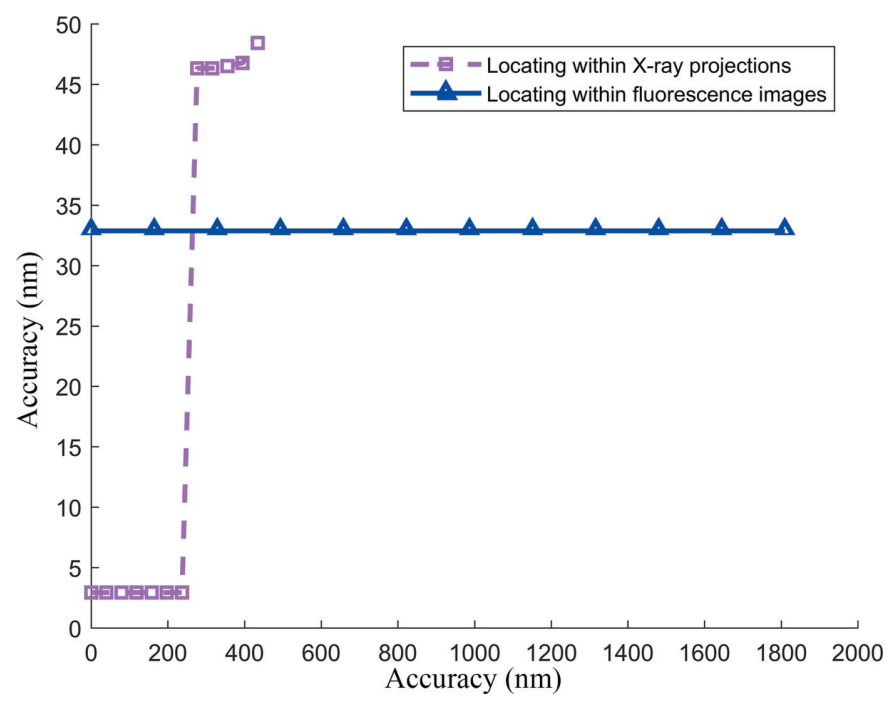

Figure 3

Relationship between the locating accuracies of the program and the distances between the initial positions and simulated true positions. The purple lines with squares and blue lines with triangles represent the locating results for X-ray projections and fluorescence images, respectively.
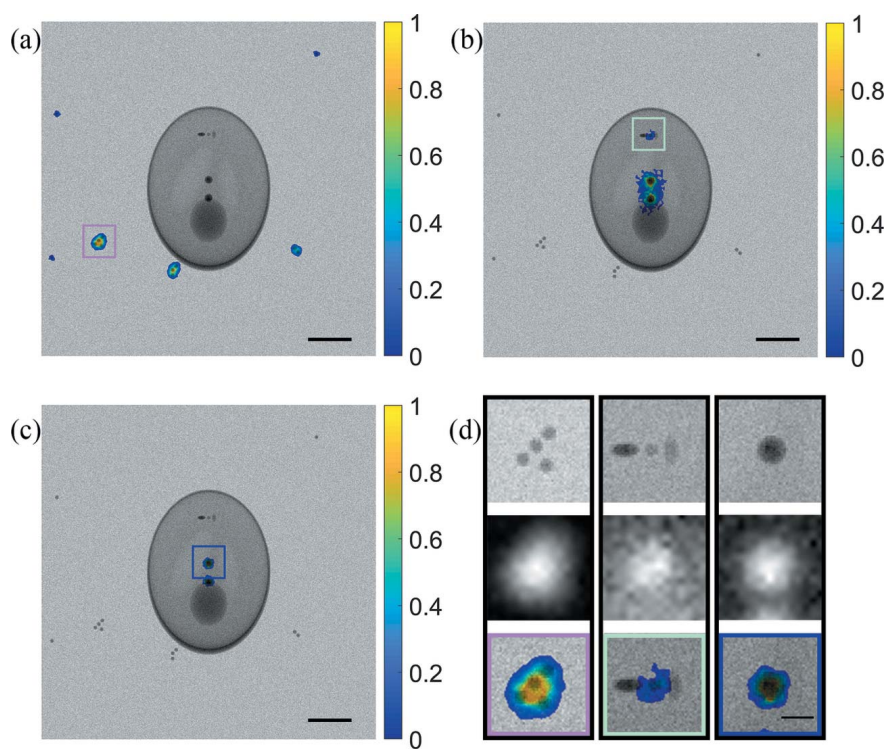

Figure 4

Locating results of the simulation. Simulated fused images are shown in $(a),(b)$ and $(c)$. Different colors indicate the intensity of normalized fluorescence. Enlargements of parts of them are shown at the bottom of (d). Small images at the top and in the middle of $(d)$ are the corresponding soft X-ray projections and fluorescence images, respectively. Scale bars: $(a),(b),(c): 2 \mu \mathrm{m} ;(d): 500 \mathrm{~nm}$.

$40 \mathrm{~nm}$ (Fig. 4). The stained object in the X-ray projection was observed to be well located by fluorescence.

\subsection{Practical experiment}

To verify the correlative method of Cryo-SXT and CryoFM, Schizosaccharomyces pombe yeasts were chosen for imaging. The yeasts were harvested and washed with water three times. Then they were stained with 5-chloromethylfluorescein diacetate (CMFDA; Molecular Probes, catalog No. C7025, Invitrogen, China) to a final concentration of $5 \mu M$ in water at $30^{\circ} \mathrm{C}$ for $45 \mathrm{~min}$. Cells were then washed with water three times to remove excess fluorescent dyes.

A standard electron microscopy grid was used to hold the cells. Before adding the cell suspension, the grid (formvar films coated coordinate copper grids, catalog No. AG200F4, Beijing Zhongjingkeyi Technology, China) was bedewed with a hydrophilic solution (ZXL-CQS solution, Laiyang Zixilai Environmental Protection Technology) to improve its hydrophilic properties. Then, a drop of the solution was spread evenly across the entire grid. Subsequently, $0.4 \mu \mathrm{l}$ of the solution with fluorescence spheres was added directly onto the grid.

There are two types of fluorescence sphere: (i) FluoSpheres [carboxylate-modified polystyrene microspheres, red fluorescent proprietary dye, excitation/emission: $580 / 605 \mathrm{~nm}, 2 \%$ solids, catalog No. F-8810, Invitrogen, China], which have red fluorescence and higher brightness; (ii) TetraSpeck spheres [0.2 $\mu \mathrm{m}$ diameter, showing four well separated excitation/ emission peaks, $360 / 430 \mathrm{~nm}$ (blue), 505/515 nm (green), 560/ $580 \mathrm{~nm}$ (orange) and 660/680 nm (dark red), Invitrogen], with 
four different fluorescences and can be suited to match with cells stained by different fluorescence.

After the grid was dried, $0.4 \mu \mathrm{l}$ of cell suspension at a cell density of $10^{8} \mathrm{ml}^{-1}$ was dropped onto it. After waiting for about $90 \mathrm{~s}$ at room temperature to evaporate some of the water to obtain a solution layer of suitable thickness, the grid was rapidly frozen by plunging into liquid ethane. Then, it was put into the specimen holder for Cryo-FM.

Independent of the cryogenic soft X-ray microscopy (Cryo-SXM), the cryogenic fluorescence microscopy assembly involves a cryo-stage (HCS302G Gas Tight Hot and Cold Stage, INSTEC) and a fluorescence microscope (Eclipse Ci, Nikon). We modified its specimen holder to fit with the standard electron microscopy grids for temporary sample transfer. A group of low-numerical-aperture air lenses were mounted on this microscope. The cryo-stage was cooled using pumped liquid nitrogen. Before the experiment, the cryo-stage should be cooled and stabilized at $-190^{\circ} \mathrm{C}$. Then, the specimen holder can be loaded into the microscope.

After the specimen holder was loaded, the rough regions of interest containing both cells and TetraSpeck microspheres were searched and collected using a low-magnification lens under bright-field and fluorescent mode. Meanwhile, the lattice coordinates were recorded for locating the rough regions of interest. Then, images at high magnification were collected using a Nikon 50×-0.6NA CFI60 TU Plan Epi ELWD objective. This microscope has three detection channels (DAPI, FITC and TRITC). Using a $50 \times$ objective, the field of view of the CCD was $6 \mathrm{~mm} \times 4.5 \mathrm{~mm}$ recorded for $1360 \times 1024$ pixels with pixel size of $88 \mathrm{~nm}$. The experiments using the FluoSpheres were performed in the TRITC (excitation: $540 / 25 \mathrm{~nm}$; emission: $605 / 55 \mathrm{~nm}$ ) channel, and the FWHM of the point spread function was $528 \mathrm{~nm}$ and the corresponding oversampling ratio was 6 . The experiments using the TetraSpeck spheres were performed in the FITC (excitation: 465-495 nm; emission: 512-558 nm) channel, and the FWHM of the point spread function was $440 \mathrm{~nm}$ and the corresponding oversampling ratio was 5 .

The cryogenic soft X-ray microscope is installed at the National Synchrotron Radiation Laboratory (NSRL), Hefei, China. To focus the X-rays, an elliptical capillary condenser is used. Coupled with a micro-zone plate, the whole system can obtain images in the absorption model with an X-ray energy ranging from $280 \mathrm{eV}$ to $700 \mathrm{eV}$ with a spatial resolution of $30 \mathrm{~nm}$. During the experiments, specimens were placed in the vacuum sample chamber cooled using liquid nitrogen.

The sample holder would be transferred to Cryo-SXM as long as data were acquired by fluorescence microscopy. Lowmagnification images were taken with an incorporated light microscope for roughly locating the same region of interest. According to the locating results, 124 high-magnification soft $\mathrm{X}$-ray projections of the same specimen with an angular range from $-57^{\circ}$ to $66^{\circ}$ were collected to match with the fluorescence images. The soft X-ray microscope was set at $520 \mathrm{eV}$ with a $15 \mu \mathrm{m} \times 15 \mu \mathrm{m}$ field of view of the CCD recorded for $950 \times 950$ pixels with a pixel size of $15.8 \mathrm{~nm}$, and the exposure time was $2 \mathrm{~s}$. Then, these projections were used to calculate a
3D reconstruction of the specimen. Each voxel in a soft X-ray tomography $3 \mathrm{D}$ reconstruction is a direct measurement of the $\mathrm{X}$-ray LAC at the corresponding location in the cell, representing the density of biomolecules. Vacuoles that have relatively high water content and membrane structure are easily distinguished from the surrounding environment. Combined with fluorescence location information, vacuoles were distinguished from other organelles, segmented from 3D tomography data using the software Amira and then rendered using this software.

To further verify the feasibility of the correlative method, rat skin mast cells were cultured for experiments. Mitochondria in the mast cells were observed by this method. A nickel grid (formvar films coated nickel grids, catalog No. AG100N, Beijing Zhongjingkeyi Technology, China) was coated with polylysine (Poly D-lysine Solution, catalog No. E607014, Sangon Biotech, China). Then, $0.2 \mu$ of solution with TetraSpeck spheres was added directly onto the grid. After the grid was dried, mast cells were allowed to attach for $10 \mathrm{~h}$. Cells were then washed by PBS and stained with MitoTracker (MitoTracker, excitation/emission: 554/576 nm, Catalog No. M7510, Invitrogen, China) to a final concentration of $10 \mathrm{n} M$ in PBS at $37^{\circ} \mathrm{C}$ for $15 \mathrm{~min}$ and washed with PBS to remove excess fluorescent dyes. Fluorescence images of the cells were obtained using the $50 \times$ objective in the TRITC channel at room temperature. Then the cells were rapidly frozen and transferred to the cryogenic soft X-ray microscope to be imaged.

\section{Results}

Using the correlative method, we imaged a number of samples under different conditions. Fig. 5 shows these fluorescence images, soft X-ray projections and fused images; the locating accuracies are shown in Table 1.

The overall uncertainties $\sigma_{\mathrm{T} 1}$ and $\sigma_{\mathrm{T} 2}$ of the sample containing dry FluoSpheres were $\sim 52 \mathrm{~nm}$ and $37 \mathrm{~nm}$, respectively, which is good enough to align the fluorescence images and soft X-ray projections. The overall uncertainties of the sample containing FluoSpheres within ice were worse than those of the sample containing dry FluoSpheres, because the fiducial markers were not distributed in the same focal plane, the fluorescent signal was attenuated due to passing through the ice layer, and the noise was much larger. The overall uncertainties $\sigma_{\mathrm{T} 1}$ and $\sigma_{\mathrm{T} 2}$ of the sample containing TetraSpeck microspheres and cells within ice were about $117 \mathrm{~nm}$ and $54 \mathrm{~nm}$. These values were better than $200 \mathrm{~nm}$, which demonstrates that our method could match fluorescence images with $\mathrm{X}$-ray images with high precision.

However, to locate vacuoles by $2 \mathrm{D}$ fluorescence images and soft X-ray tomography images, fluorescence images with different focal lengths need to be fused. Vacuoles, which were stained with CMFDA, were always not in the same focal plane as the fiducial markers, which led to difficulties in observing the vacuoles and fiducial markers clearly in the same fluorescence images. It was difficult to distinguish each vacuole 

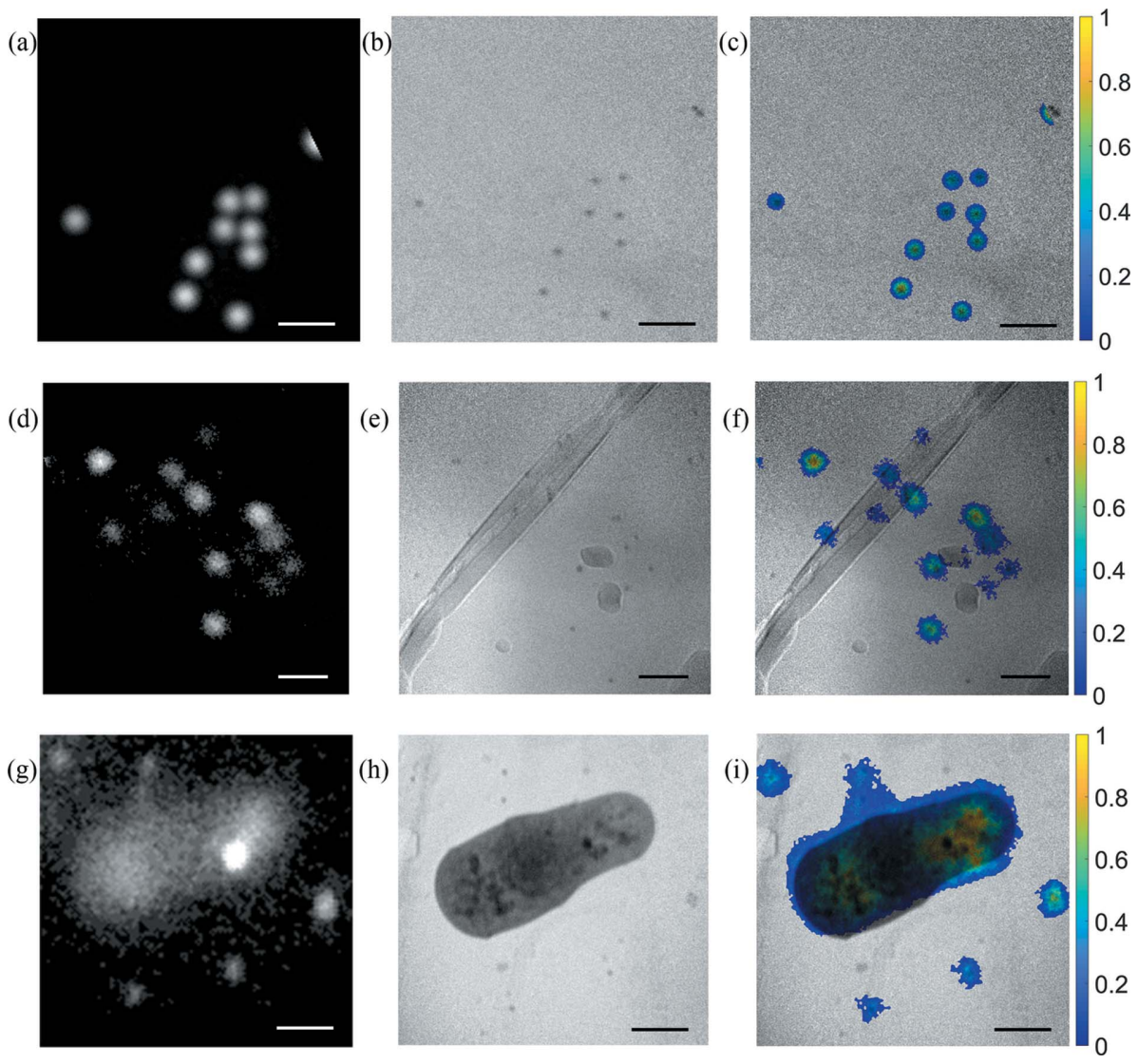

Figure 5

Fluorescence images $[(a),(d)$ and $(g)]$, soft X-ray projections $[(b),(e)$ and $(h)]$ and fused images $[(c),(f)$ and $(i)]$ of samples. Different colors in $(c),(f)$ and $(i)$ indicate the intensity of normalized fluorescence. Panels $(a),(b)$ and $(c)$ are images of the sample containing dry FluoSpheres. Panels $(d),(e)$ and $(f)$ are images of the sample containing FluoSpheres within ice. Panels $(g),(h)$ and $(i)$ are images of the sample containing TetraSpeck microspheres and cells within ice. Scale bars: $2 \mu \mathrm{m}$.

Table 1

Accuracies in the alignment process of different samples.

\begin{tabular}{llllllllll}
\hline & $\begin{array}{l}\text { With } \\
\text { fluorescence }\end{array}$ & $\begin{array}{l}\text { Within } \\
\text { ice }\end{array}$ & $\begin{array}{l}\text { With } \\
\text { cells }\end{array}$ & $\begin{array}{l}\sigma_{\mathrm{X}} \\
(\mathrm{nm})\end{array}$ & $\begin{array}{l}\sigma_{\mathrm{F}} \\
(\mathrm{nm})\end{array}$ & \multicolumn{1}{l}{$\begin{array}{l}\sigma_{\mathrm{A} 1} \\
(\mathrm{~nm})\end{array}$} & $\begin{array}{l}\sigma_{\mathrm{A} 2} \\
(\mathrm{~nm})\end{array}$ & $\begin{array}{l}\sigma_{\mathrm{T} 1} \\
(\mathrm{~nm})\end{array}$ & $\begin{array}{l}\sigma_{\mathrm{T} 2} \\
(\mathrm{~nm})\end{array}$ \\
\hline 1 & Yes & No & No & 5.6 & 13.8 & 49.9 & 33.9 & 52.1 & 37.0 \\
2 & Yes & Yes & No & 8.7 & 16.9 & 151.4 & 88.9 & 152.6 & 90.9 \\
3 & Yes & Yes & Yes & 10.2 & 23.7 & 114.3 & 47.5 & 117.2 & 54.1 \\
\hline
\end{tabular}

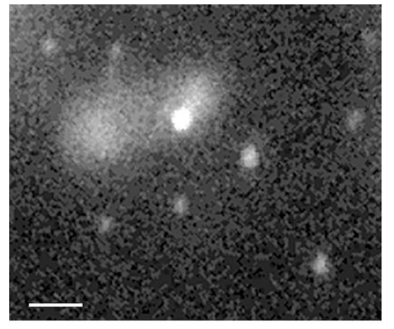

(a)

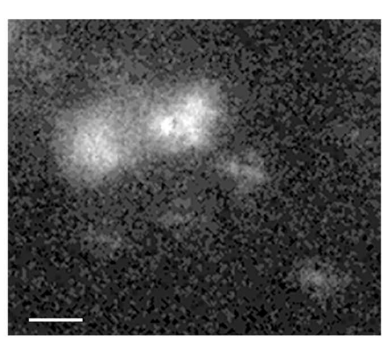

(b)

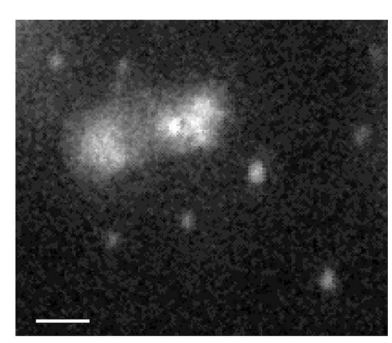

(c)
Figure 6

$(a)$ and $(b)$ Fluorescence images obtained at different focal lengths. (c) Combined image of $(a)$ and (b). Scale bars: $2 \mu \mathrm{m}$. when the fiducial markers were clear [Fig. 6(a)], and the fiducial markers were blurred when some of the vacuoles were identifiable [Fig. 6(b)]; therefore, we fused Figs. 6(a) and $6(b)$ as a combined image in which both the fiducial markers and vacuoles were clear [Fig. 6(c)].

Next, the soft X-ray projection and the combined fluorescence images were aligned and fused, as shown in Fig. 7(a). Enlargements of parts of it are shown in Fig. 7(b). The red crosses indicate the locating results of fiducial markers in the fluorescence images, and the blue crosses indicate the locating results of fiducial markers in the soft X-ray projections. The red dashed circles are centered on the red crosses with the overall error $\sigma_{\mathrm{T}}$ as the radii. All blue crosses are in the red circles, which demonstrates the feasibility of our error calculation method.

To locate the vacuoles, fluorescence images and a slice of the soft X-ray tomography image were fused as shown in Fig. 7(c). The vacuoles were stained with CMFDA and could be located by fluorescence. The structure of the vacuoles is clearly shown in the SXT slice. The small area containing the stained structure can be precisely located in the radial direction using the fluorescence signals; then, the position of the stained structure in the axial direction can be located by observing the structure corresponding to the fluorescence signals in the soft X-ray tomography image in the small area. Combining information from different modalities, four organelles whose centers are covered by fluorescence were determined as vacuoles. The segmented results of the vacuoles are shown in Figs. $7(d)$ and 8. The segmented structure with an LAC value of $0.21 \pm$ $0.03 \mu \mathrm{m}^{-1}$, which is close to the reference LAC values of $0.214 \pm 0.025 \mu \mathrm{m}^{-1}$ (Liu et al., 2018) and $0.22 \pm 0.07 \mu \mathrm{m}^{-1}$ (Uchida et al., 2011) for vacuoles, was believed to be a vacuole (see Table 2). This further demonstrated the feasibility of our correlative method to identify organelles.

Results for the mast cells are shown in Fig. 9. Mitochondria in the protuberance part of the mast cell were observed. The fluorescence image of the mitochondria and fluorescence spheres is shown in Fig. 9(a). A mosaic image of soft $\mathrm{X}$-ray projections at $0^{\circ}$ is shown 
in Fig. 9(b). These images were aligned with fluorescence spheres, and the overall uncertainties $\sigma_{\mathrm{T} 1}$ and $\sigma_{\mathrm{T} 2}$ were about $88 \mathrm{~nm}$ and $31 \mathrm{~nm}$, respectively. The fused image of the fluorescence images and the mosaic image of soft X-ray projections are shown in Fig. 9(c). The soft X-ray tomography image was obtained in the area containing bright fluorescence signals, shown in Fig. $9(d)$. The fused image of the fluorescence images and a slice of the tomography image are shown in Fig. 9(e). The rough locations of the mitochondria in the radial direction can be indicated by the wide-field fluorescence signals; the structures and precise locations of the mitochondria can be
Table 2

LAC values of vacuoles.

\begin{tabular}{|c|c|c|}
\hline $\begin{array}{l}\text { LAC values of different } \\
\text { vacuoles }\left(\mu \mathrm{m}^{-1}\right)\end{array}$ & $\begin{array}{l}\text { Average LAC } \\
\text { value }\left(\mu \mathrm{m}^{-1}\right)\end{array}$ & $\begin{array}{l}\text { Reference LAC } \\
\text { values }\left(\mu \mathrm{m}^{-1}\right)\end{array}$ \\
\hline $\begin{array}{l}0.184 \\
0.222 \\
0.245 \\
0.206\end{array}$ & $0.214 \pm 0.03$ & $\begin{array}{l}0.214 \pm 0.025 \\
\text { (Liu et al., 2018) } \\
0.22 \pm 0.07 \\
\text { (Uchida } \text { et al., 2011) }\end{array}$ \\
\hline
\end{tabular}

indicated by the slices of the tomography image. The mitochondria located by our method were segmented and shown in Figs. $9(f)$ and $9(g)$. (a)

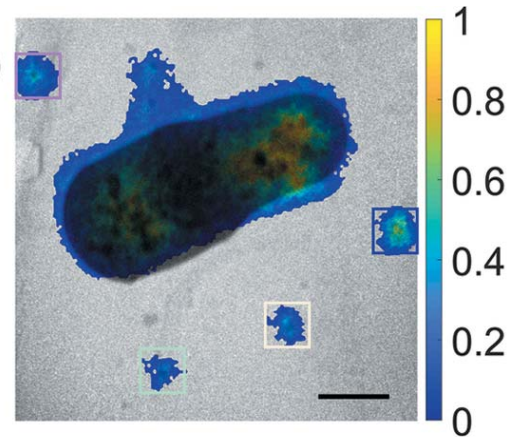

(c)

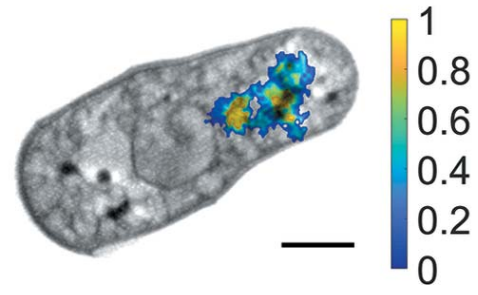

(b)
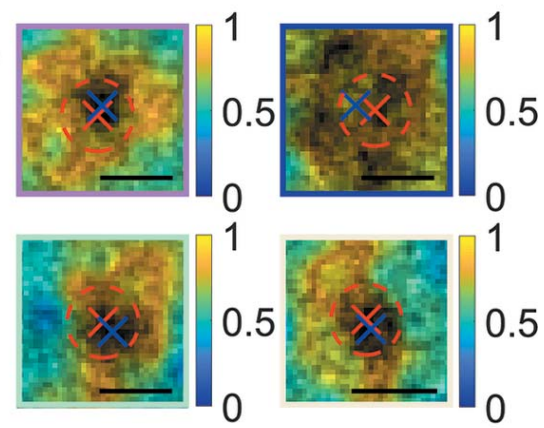

(d)

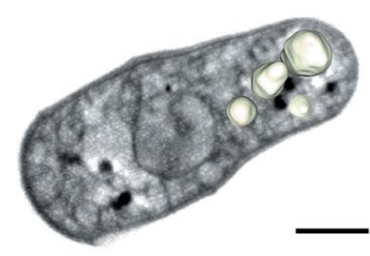

Figure 7

Locating vacuoles by fluorescence and X-ray images. (a) Fused image of the fluorescence image and the X-ray projection. Fiducial markers are enlarged and shown in $(b)$. Blue crosses mark the locating result of the fiducial markers in the X-ray projection, and red crosses mark the corresponding fiducial markers in the fluorescence image. (c) Fused image of the fluorescence image and a slice of the soft X-ray tomography image. Different colors in $(a),(b)$ and $(c)$ indicate the intensity of normalized fluorescence. $(d)$ Segmentations of vacuoles. Scale bars: $(a),(c),(d): 2 \mu \mathrm{m}$; (b): $250 \mathrm{~nm}$.

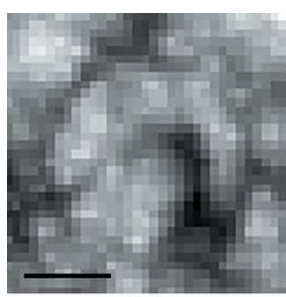

(a)

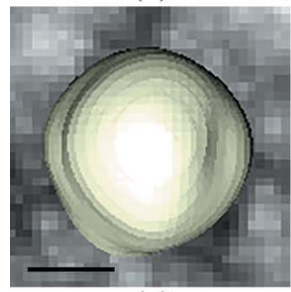

(e)

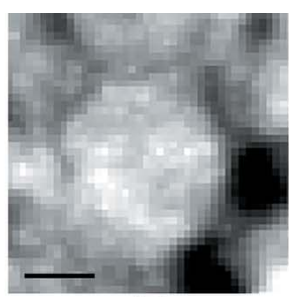

(b)

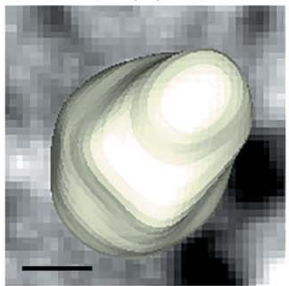

(f)

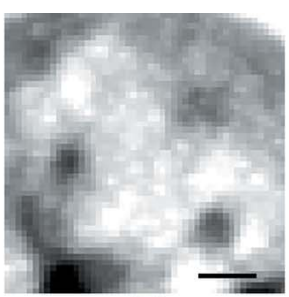

(c)

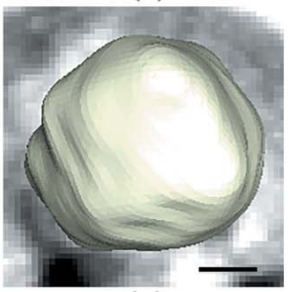

(g)

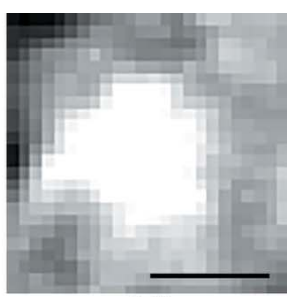

(d)

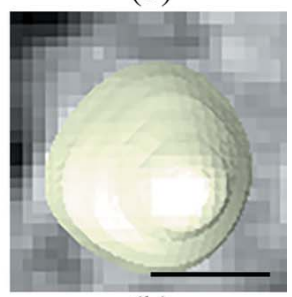

(h)

Figure 8

Slices $[(a)-(d)]$ of soft X-ray tomography of vacuoles and their segments $[(e)-(h)]$. Scale bars: $300 \mathrm{~nm}$.

\section{Conclusions}

In summary, we introduce a precise alignment and fusion method of CryoSXT and Cryo-FM and demonstrate the feasibility of the proposed method via simulations and experiments. Our method can reduce subjective participation and enhance alignment accuracy. Although the method involves initial manual operation, the simulations indicate that the result was almost uninfluenced by manual operation. The method showed stable performance when some fluorescence spheres agglomerated, a situation that often occurs. The total alignment accuracies were worse when samples were in ice compared with dry samples, but they were all better than $200 \mathrm{~nm}$. This means that we could locate the sub-cellular architecture in soft X-ray images using corresponding fluorescence images with high accuracy.

Using this method, we located the vacuoles of yeast and the mitochondria of a mast cell with fluorescence images and SXT images. This demonstrates that our result is credible, and our method is feasible for the recognition and classification of organelles.

A limitation is that wide-field fluorescence will be mixed if some stained objects are close together. Large subcellular structures can be located in a thick cellular volume using our method, and small sub-cellular structures can only be located in a thin cellular volume. We choose fluorescence of the highest brightness to locate objects, but this still causes confusion sometimes and extra experiments are required. Another problem is that wide-field fluorescence microscopy cannot provide an accurate 


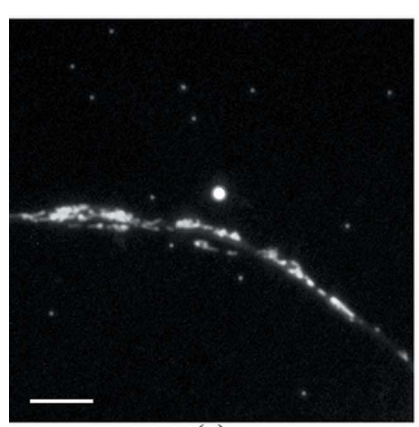

(a)

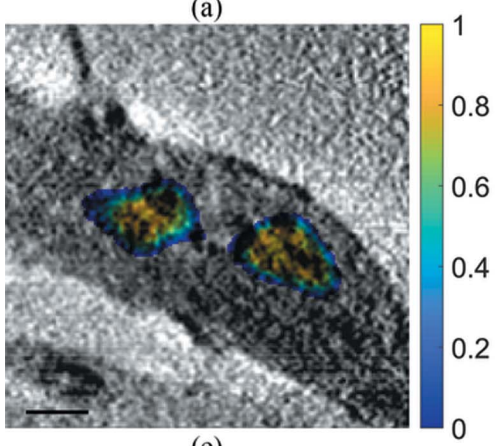

(e)

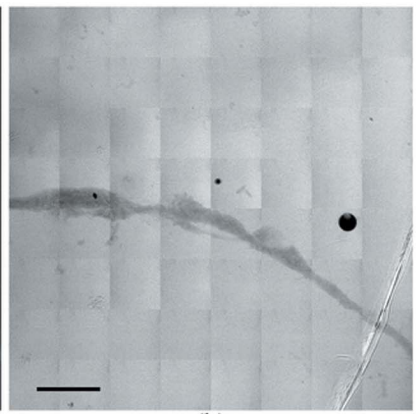

(b)

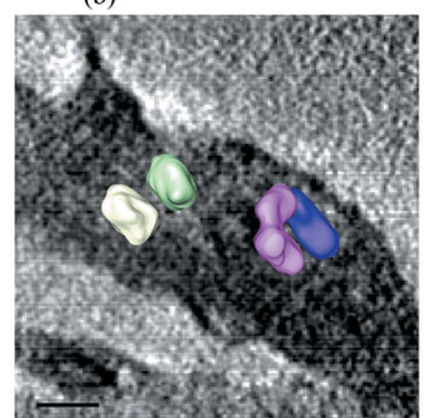

(f)

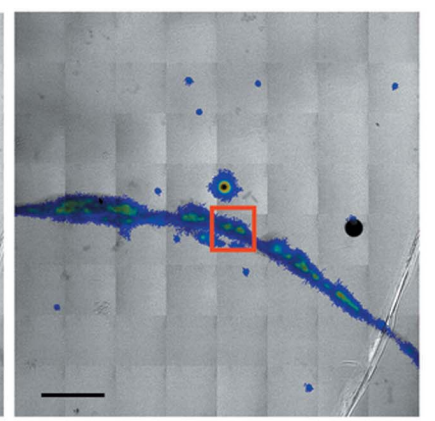

(c)
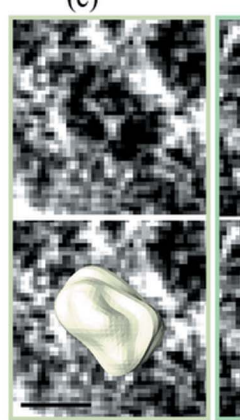

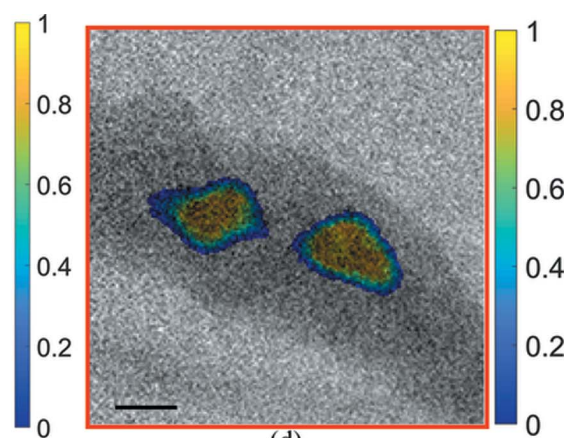

(d)

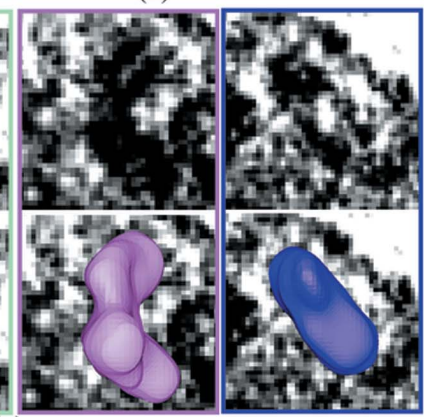

(g)

Figure 9

Correlative imaging of mitochondria of the mast cell. (a) Fluorescence image of the protuberance part of the mast cell, (b) mosaic image of soft X-ray projections at $0^{\circ}$ of the same part of the mast cell and $(c)$ fused image of $(a)$ and $(b)$. An enlargement of the area surrounded by the red square in $(c)$ is shown in $(d)$. (e) Fused image of the fluorescence image and a slice of the soft X-ray tomography image. $(f)$ Segmentations of mitochondria. $(g)$ Enlargements of the segmentations of mitochondria and corresponding soft X-ray tomography slices. The different colors in $(c),(d)$ and $(e)$ indicate the intensity of normalized fluorescence. Scale bars: $(a),(b),(c): 10 \mu \mathrm{m} ;(d),(e),(f): 1 \mu \mathrm{m} ;(g): 500 \mathrm{~nm}$.

position in the direction of the optical axis. A small area containing the stained structure can be precisely located in the radial direction. But the stained structure has to be searched in a rough range in the axial direction in a soft X-ray tomography image, for which the number of similar structures at different axial distances in the small area should be as small as possible. More experiments are needed to satisfy this requirement. Using 3D fluorescence imaging technology, such as confocal fluorescence microscopy and super-resolution fluorescence microscopy, could help resolve this problem.

\section{Acknowledgements}

We would like to thank Wenyue Liu from Dr Chuanhai Fu's laboratory for support in the cell culture growth and maintenance.

\section{Funding information}

The research leading to these results has received funding from the National Key Research and Development Program of China (grant Nos 2017YFA0402904; 2016YFA0400902) and the National Natural Science Foundation of China (NSFC) (grant Nos 11775224; 11475175)

\section{References}

Carzaniga, R., Domart, M.-C., Collinson, L. M. \& Duke, E. (2014). Protoplasma, 251, 449-458.
Chao, W. L., Harteneck, B. D., Liddle, J. A., Anderson, E. H. \& Attwood, D. T. (2005). Nature, 435, 1210-1213.

Cheezum, M. K., Walker, W. F. \& Guilford, W. H. (2001). Biophys. J. 81, 2378-2388.

Cinquin, B. P., Do, M., McDermott, G., Walters, A. D., Myllys, M., Smith, E. A., Cohen-Fix, O., Le Gros, M. A. \& Larabell, C. A. (2014). J. Cell. Biochem. 115, 209-216.

Dent, K. C., Hagen, C. \& Gruenewald, K. (2014). Methods Cell Biol. 124, 179-216.

Do, M., Isaacson, S. A., McDermott, G., Le Gros, M. A. \& Larabell, C. A. (2015). Arch. Biochem. Biophys. 581, 111-121.

Duke, E. M. H., Razi, M., Weston, A., Guttmann, P., Werner, S., Henzler, K., Schneider, G., Tooze, S. A. \& Collinson, L. M. (2014). Ultramicroscopy, 143, 77-87.

Ekman, A. A., Chen, J. H., Guo, J., McDermott, G., Le Gros, M. A. \& Larabell, C. A. (2017). Biol. Cell, 109, 24-38.

Elgass, K. D., Smith, E. A., LeGros, M. A., Larabell, C. A. \& Ryan, M. T. (2015). J. Cell Sci. 128, 2795-2804.

Hagen, C., Guttmann, P., Klupp, B., Werner, S., Rehbein, S., Mettenleiter, T. C., Schneider, G. \& Grünewald, K. (2012). J. Struct. Biol. 177, 193-201.

Larabell, C. A. \& Le Gros, M. A. (2004). Mol. Biol. Cell, 15, 957962.

Li, J. Z., Xue, F. \& Blu, T. (2017). J. Opt. Soc. Am. A Opt. Image Sci. Vis. 34, 1029-1034.

Liu, J., Li, F., Chen, L., Guan, Y., Tian, L., Xiong, Y., Liu, G. \& Tian, Y. (2018). J. Microsc. 270, 64-70.

McDermott, G., Le Gros, M. A., Knoechel, C. G., Uchida, M. \& Larabell, C. A. (2009). Trends Cell Biol. 19, 587-595.

McDermott, G., Le Gros, M. A. \& Larabell, C. A. (2012). Annu. Rev. Phys. Chem. 63, 225-239.

Mohammadian, S., Fokkema, J., Agronskaia, A. V., Liv, N., de Heus, C., van Donselaar, E., Blab, G. A., Klumperman, J. \& Gerritsen, H. C. (2019). Sci. Rep. 9, 10. 
Schellenberger, P., Kaufmann, R., Siebert, C. A., Hagen, C., Wodrich, H. \& Grünewald, K. (2014). Ultramicroscopy, 143, 41-51.

Schneider, G. (1998). Ultramicroscopy, 75, 85-104.

Smith, E. A., Cinquin, B. P., Do, M., McDermott, G., Le Gros, M. A. \& Larabell, C. A. (2014). Ultramicroscopy, 143, 33-40.

Smith, E. A., Cinquin, B. P., McDermott, G., Le Gros, M. A., Parkinson, D. Y., Kim, H. T. \& Larabell, C. A. (2013). J. Struct. Biol. 184, 12-20.

Smith, E. A., McDermott, G., Do, M., Leung, K., Panning, B., Le Gros, M. A. \& Larabell, C. A. (2014). Biophys. J. 107, 1988-1996.

Sorrentino, A., Nicolás, J., Valcárcel, R., Chichón, F. J., Rosanes, M., Avila, J., Tkachuk, A., Irwin, J., Ferrer, S. \& Pereiro, E. (2015). J. Synchrotron Rad. 22, 1112-1117.

Thompson, R. E., Larson, D. R. \& Webb, W. W. (2002). Biophys. J. 82, 2775-2783.
Uchida, M., Sun, Y., McDermott, G., Knoechel, C., Le Gros, M. A., Parkinson, D., Drubin, D. G. \& Larabell, C. A. (2011). Yeast, 28, 227-236.

Varsano, N., Beghi, F., Elad, N., Pereiro, E., Dadosh, T., Pinkas, I., Perez-Berna, A. J., Jin, X. T., Kruth, H. S., Leiserowitz, L. \& Addadi, L. (2018). Proc. Natl Acad. Sci. USA, 115, 7662-7669.

Varsano, N., Dadosh, T., Kapishnikov, S., Pereiro, E., Shimoni, E., Jin, X. T., Kruth, H. S., Leiserowitz, L. \& Addadi, L. (2016). J. Am. Chem. Soc. 138, 14931-14940.

Walters, A. D., May, C. K., Dauster, E. S., Cinquin, B. P., Smith, E. A., Robellet, X., D'Amours, D., Larabell, C. A. \& Cohen-Fix, O. (2014). Curr. Biol. 24, 2861-2867.

Wolter, H. (1952). Ann. Phys. 445, 94-114.

Zeev-Ben-Mordehai, T., Hagen, C. \& Grünewald, K. (2014). Curr. Opin. Virol. 5, 42-49. 